\title{
Spitzer Space Telescope: Dark Current and Total Noise Prediction for InSb Detector Arrays in the Infrared Array Camera (IRAC) for the Post-Cryogen Era
}

\author{
Craig W. McMurtry, Judith L. Pipher and William J. Forrest \\ University of Rochester, Rochester, NY, USA
}

\begin{abstract}
During the expected $5+$ years of operation, the Spitzer Space Telescope is and will continue to produce outstanding infrared images and spectra, and greatly further scientific understanding of our universe. The Spitzer Space Telescope's instruments are cryogenically cooled to achieve low dark current and low noise. After the cryogens are exhausted, the Spitzer Space Telescope will only be cooled by passively radiating into space. The detector arrays in the IRAC instrument are expected to equilibrate at approximately $30 \mathrm{~K}$. The two shortest wavelength channels (3.6 and 4.5 micron) employ InSb detector arrays and are expected to function and perform with only a modest degradation in sensitivity. Thus, an extended mission is possible for Spitzer. We present the predicted dark current, noise, quantum efficiency and image residuals for the 3.6 and 4.5 micron IRAC channels in the post-cryogen era.
\end{abstract}

Keywords: Spitzer Space Telescope, IRAC, InSb, cryogen, passive cooling, infrared, dark current, residual images

\section{INTRODUCTION}

The InSb detector arrays on the Infrared Array Camera (IRAC) are currently operating at a temperature of $15.0 \mathrm{~K}$ and are cooled via a liquid helium cryostat. This paper details initial testing to determine the feasibility of continued operation of both of the InSb detector arrays (IRAC Channels 1 and 2) after cryogens are exhausted on the Spitzer Space Telescope. From thermal models and calculations, current estimates indicate that the final temperatures of the Channel 1 and 2 detector arrays will be between $25 \mathrm{~K}$ and $31 \mathrm{~K}$, of course, dependent upon the power dissipation of the detector arrays and the thermal characteristics of the flight mounts at those temperatures. Where applicable, we will compare previous test results at $\mathrm{T}=15.0 \mathrm{~K}$ with new results obtained at or near $\mathrm{T}=30 \mathrm{~K}$. We refer the reader to published works regarding development and operation of the telescope, instrument and detector arrays. . $^{1,3,4,5,6}$

Of the four IRAC Sensor Chip Assemblies (SCAs 48536, 48537, 48538, and 48540) in flight mounted Focal Plane Assemblies (FPAs) available for post-cryogen testing in our laboratory, we chose SCA 48538 based upon: 1) its ROIC and detector array similarities with Channel 1 and, in part, Channel 2 and 2) the extensive prior testing of the SCA at $\mathrm{T}=15 \mathrm{~K}$ by the University of Rochester. Tests were conducted with SCA 48538 (a.k.a. FPA 37, full IRAC flight mounted array), which has a Read-Out Integrated Circuit (ROIC or multiplexer) from the same lot split (Lot 1, from Lawrence Semiconductor) as both Channel 1 (SCA 48534, FPA 34) and Channel 2 (SCA 48975, FPA 66). SCA 48538 has a detector array from the same InSb boule (although different wafer) as Channel 1. However the detector array material for Channel 2 came from a different vendor (Firebird). Since the processing done on the detector starting material by Raytheon (SBRC) determines many of the relevant properties, we expect only small deviations in quantum efficiency and dark current between the two channels.

\section{DATA}

\subsection{Clocks and Biases}

Operating parameters (clocks and biases) are provided in Table 1. Test procedures are detailed by Wu et al. ${ }^{7}$ Of the eight clocks and eleven biases, only the bias Vgg1 needed adjust for proper operation at $\mathrm{T}=30 \mathrm{~K}$. The IRAC electronics have adjustable D/A converters for four biases, namely Vgg1, Vdduc, Vrstuc and Vdet, while the remainder of the biases and clocks have fixed voltage levels.

For further information, E-mail: craig.mcmurtry@rochester.edu 
Table 1. Voltages used for clocks and biases with SCA 48538 operating near $\mathrm{T}=30 \mathrm{~K}$.

\begin{tabular}{lcc} 
Clock & Value (Volts) & $\begin{array}{c}\text { Changed from } \\
\text { 15K Operation? }\end{array}$ \\
\hline \hline$\phi$ Rst & $-3.0 /-5.5$ & No \\
$\phi$ ggcl & $-1.0 /-5.0$ & No \\
\hline$\phi 1 \mathrm{~F}$ & $-1.0 /-5.0$ & No \\
$\phi 2 \mathrm{~F}$ & $-1.0 /-5.0$ & No \\
$\phi$ SyncF & $-1.0 /-5.0$ & No \\
\hline$\phi 1 \mathrm{~S}$ & $-1.0 /-7.0$ & No \\
$\phi 2 \mathrm{~S}$ & $-1.0 /-7.0$ & No \\
$\phi$ SyncS & $-1.0 /-7.0$ & No \\
\hline
\end{tabular}

\begin{tabular}{lccc} 
Bias & Value (Volts) & $\begin{array}{c}\text { Changed from } \\
\text { 15K Operation? }\end{array}$ & $\begin{array}{c}\text { IRAC } \\
\text { Adjustable? }\end{array}$ \\
\hline \hline Vgg1 & $-2.37^{*}$ & Yes* & Yes \\
Vgg2 & 0.0 & No & No \\
Vdduc & -3.5 & No & Yes \\
\hline VssScanS & -1.0 & No & No \\
VssScanF & -1.0 & No & No \\
Vddcl & -1.9 & No & No \\
\hline Vddout & -1.2 & No & No \\
Vss1 & -1.0 & No & No \\
Vss2 & -1.0 & No & No \\
\hline Vrstuc & -3.50 & No & Yes \\
VDet & $-3.05^{\dagger}$ & No & Yes \\
\hline
\end{tabular}

* We set Vgg1 such that the current between Vdduc and Vss1 was $100 \mu A(\operatorname{Vgg} 1=-2.37 \mathrm{~V})$.

$\dagger$ The applied detector reverse bias was $450 \mathrm{mV}$, i.e. Vrstuc - VDet.

Table 2. Temperatures of the UR baseplate and SCA 48538 obtained during testing.

\begin{tabular}{l|cccccccccc} 
& \multicolumn{10}{|c}{ Temperature (K) } \\
\hline \hline UR Baseplate & 9.9 & 11.0 & 12.9 & 27.9 & 29.1 & 29.6 & 31.3 & 33.0 & 34.6 & 36.9 \\
SCA & 12.6 & 13.6 & 15.2 & 29.2 & 30.1 & 30.5 & 32.3 & 34.0 & 35.5 & 37.6 \\
\hline
\end{tabular}

\subsection{Temperature, Thermal Conductivity and Power Dissipation}

The focus of the tests conducted at the University of Rochester is the detector array performance and not the cryogenic thermal properties of the flight arrays operating at the anticipated final temperatures. As such, our mount is not meant to mimic the FPA mounts in IRAC, but instead provides a baseplate which is operated at a given temperature. Nevertheless, we detail relevant information pertaining to the thermal properties of the FPA as operated in our system, which will further inform the Spitzer Space Telescope cryogenic engineers and allow for detailed predictions of SCA final temperatures in the post cryogen era.

Initially, the power to the array was left off. After stabilization of the University of Rochester's baseplate at $\mathrm{T}=28 \mathrm{~K}$, we turned on the power to SCA 48538 , but did not use the heaters present on-board the SCA. The detector array was then allowed to further stabilize in temperature, without active temperature control for either the baseplate or the SCA. The final baseplate temperature was $\mathrm{T}=29.6 \mathrm{~K}$, and the final SCA temperature was $\mathrm{T}=30.5 \mathrm{~K}$. All baseplate and associated SCA temperatures used during testing are listed in Table 2 . 


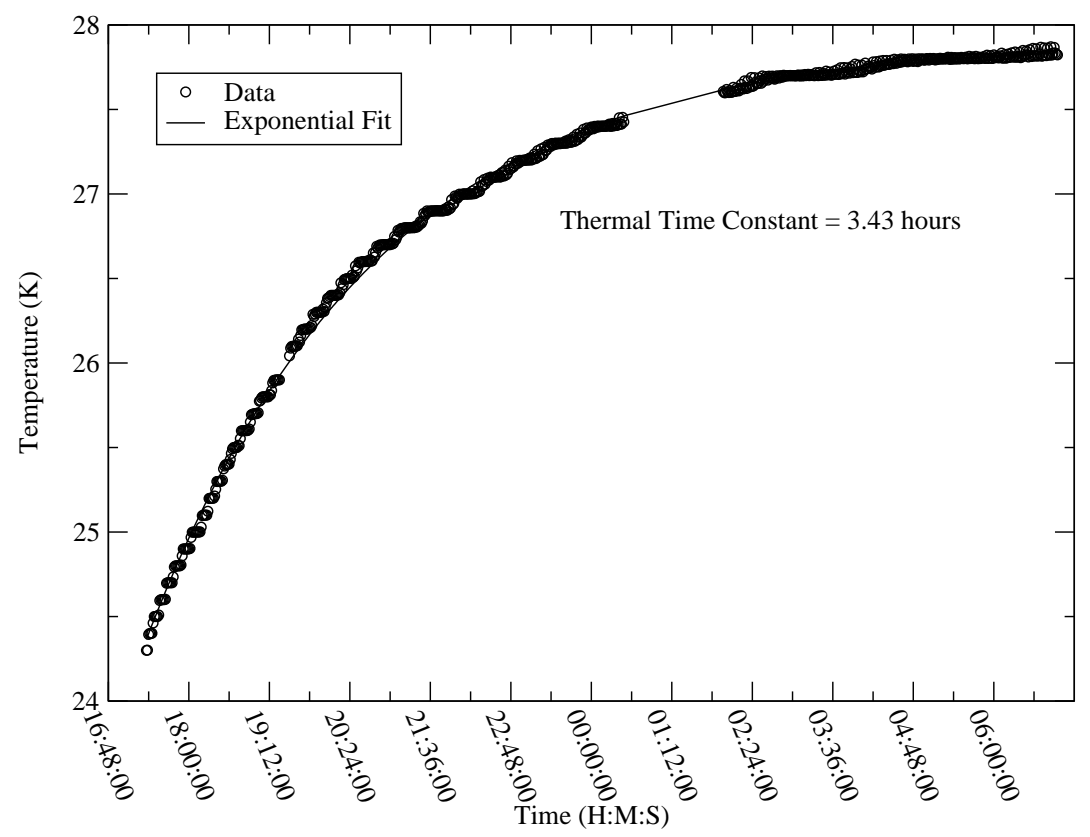

Figure 1. This graphs shows temperature versus time data for the flight mounted SCA 48538. These data represent the thermal time constant of the entire flight mount, our mount plate, mount support structures and cables. The small periodic fluctuations in temperature are due to digitization error in reading the University of Rochester's baseplate temperature sensor.

The temperature difference between the University of Rochester's baseplate with the detector array off versus on is related to the thermal conductivity of our mount that connects the baseplate to the LHe reservoir, and is not a direct measurement of the SCA thermal conductivity. Over the course of our experiments, we varied the temperature of the baseplate and measured the time constant of our full system (flight mounted SCA, baseplate and mount structures). The thermal time constant of our complete system is quite large $(\tau=3.43 \mathrm{hr}$, see Figure 1). While this large thermal time constant adds significant overhead to all of our laboratory measurements, it also allows for an extremely temperature stable SCA without active temperature control of either the baseplate or the SCA.

In order to measure the thermal time constant of the SCA (InSb detector array and ROIC) with its motherboard and exclude the thermal time constant of our complete system, we used both the temperature sensing diode and the heater resistor on the SCA motherboard. After the University of Rochester's baseplate had reached a stabilized temperature, we turned on the SCA heater and allowed the SCA (and complete system) to equilibrate. The thermal time constant of just the SCA and its motherboard is 2.50 minutes when operating near $15 \mathrm{~K}$ (see Figure 2) and 4.20 minutes at temperatures near 28K (see Figure 3). With an initial SCA temperature of 13.6K and SCA heater power of $0.35 \mathrm{~mW}$, the final temperature reached was $16.0 \mathrm{~K}$, where the final SCA temperature is given by the time constant fits and not by the continually increasing temperature seen in the data, which is discussed in more detail below. Similarly, with an initial SCA temperature of $26.8 \mathrm{~K}$, the SCA heater power was $0.78 \mathrm{~mW}$ to get to the final SCA temperature of $29.2 \mathrm{~K}$. While we attempted to decouple the longer thermal time constant of the complete system, there is still evidence of the longer thermal time constant (cf. Figure 1) in the gradually rising temperature data above the exponential fit in both Figures 2 and 3. The Si diode temperature sensor on the SCA motherboard exhibited Random Telegraph Signal (RTS) noise (also known as burst noise or popcorn noise, see Bacon et $a l^{8}$ ) as evidenced by the dual level nature of the data in Figure 3. The RTS noise is 


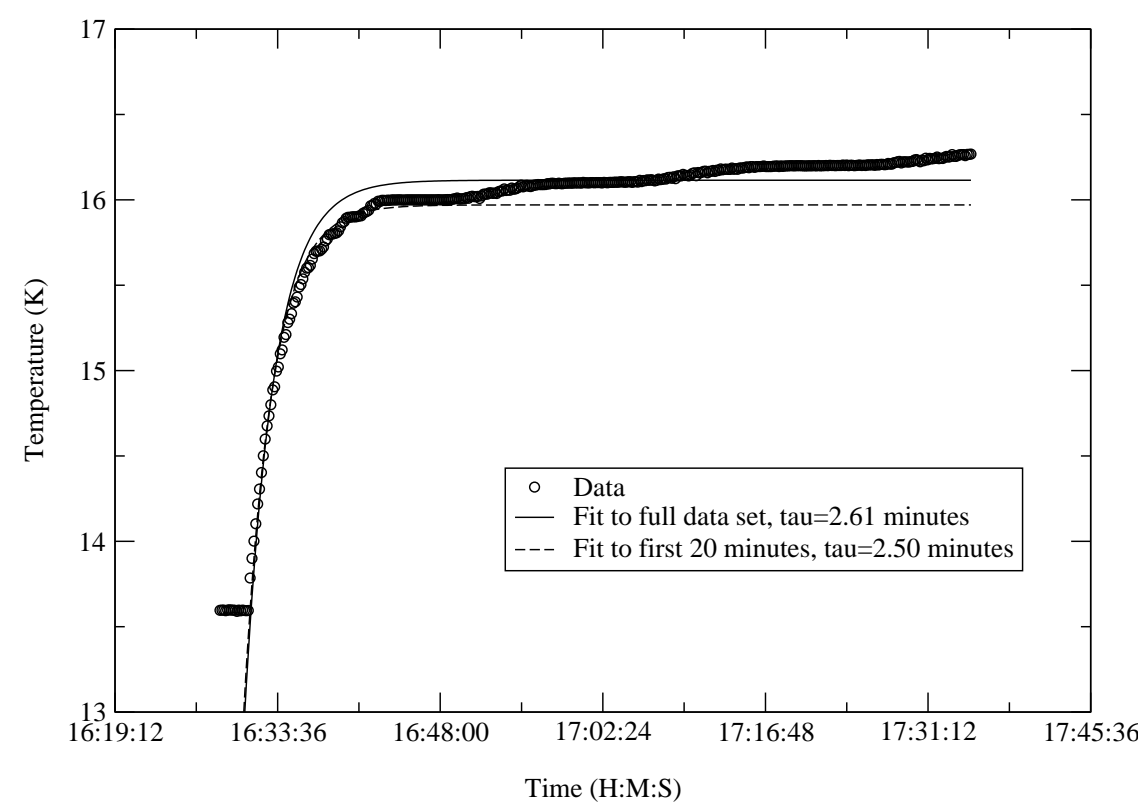

Figure 2. This graph shows temperature versus time data for the flight mounted SCA 48538. These data (mainly) represent the thermal time constant of just the SCA (InSb detector array and ROIC) with its motherboard around $\mathrm{T}=15 \mathrm{~K}$.

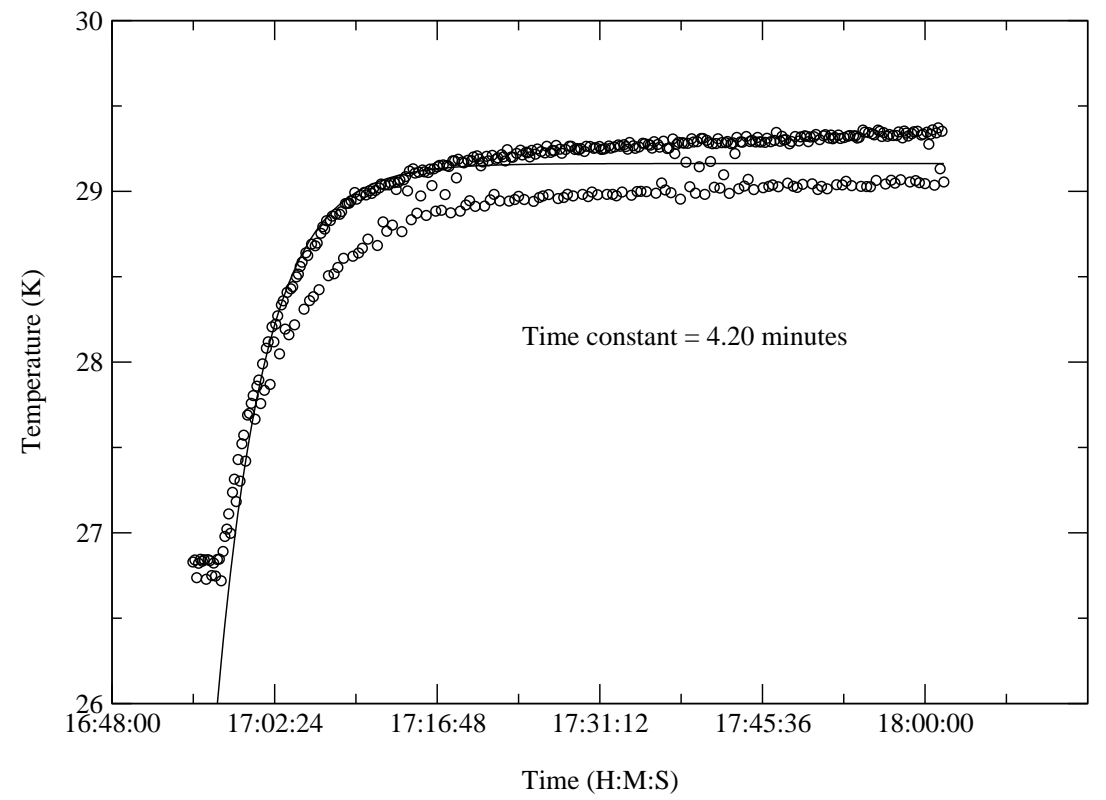

Figure 3. This graph shows temperature versus time data for the flight mounted SCA 48538. These data (mainly) represent the thermal time constant of just the SCA (InSb detector array and ROIC) with its motherboard around $\mathrm{T}=28 \mathrm{~K}$. There are two issues discussed in the text: dual level nature of the data and continuing increase in temperature above the exponential fit. The higher temperature curve is the correct temperature, while the lower is offset due to a voltage shift. 
seen in only this small Si diode temperature sensor on the SCA motherboard and not in any of the larger Si diode temperature sensors employed by the University of Rochester or in the cernox temperature sensors. Further, the RTS noise seen in Figure 3 is significantly above the digitization error seen in Figure 1, which are data from a different temperature sensor. The Si diode temperature sensor on the SCA motherboard demonstrates RTS noise at temperatures near $30 \mathrm{~K}$, but not at temperatures below $16 \mathrm{~K}$, indicating a "turn-on" temperature for the trap induced RTS noise, which has not been investigated further. The RTS noise in this Si diode temperature sensor is neither an issue for our measurements, since the diode has a dominant level which tends to be sampled, nor for Spitzer, since the temperature is measured using the cernox temperature sensors.

Using calorimetric methods, we measured the power dissipation of SCA 48538 to be $0.3 \mathrm{~mW}$ at $\mathrm{T}=30 \mathrm{~K}$, although the uncertainty is $-0.1 /+0.15 \mathrm{~mW}$ due to the large thermal time constants of our setup (see above). The power dissipation of SCA 48538 at $\mathrm{T}=15 \mathrm{~K}$ was $0.50 \mathrm{~mW}$. For comparison, the two IRAC InSb arrays were measured to dissipate $0.40 \mathrm{~mW}$ at $\mathrm{T}=15 \mathrm{~K}$.

\subsection{Laboratory Calibration}

We measured the source follower gain of the ROIC to be 0.87 , which represents no change between $\mathrm{T}=15.0 \mathrm{~K}$ and $\mathrm{T}=30.4 \mathrm{~K}$ operation.

The nodal capacitance was measured using the noise squared versus signal method and is $67.2 \mathrm{fF}$, which is also unchanged from operation at $\mathrm{T}=15 \mathrm{~K}$. For the applied detector reverse bias of $450 \mathrm{mV}$, the actual well depth is $147,000 e^{-}$.

With a $600 \mathrm{pF}$ load attached to each output, the FPA output voltage has a rising time constant of $1.3 \mu s$ and a falling time constant of $1.1 \mu \mathrm{s}$. The FPAs can easily drive the IRAC load at $\mathrm{T}=30 \mathrm{~K}$, i.e. greater than 7 time constants in the $10 \mu s$ pixel dwell time.

\subsection{Dark Current}

As a function of temperature, we measured the dark current for SCA 48538 at an applied detector reverse bias of $450 \mathrm{mV}$ (see Figure 4). We employed a darkened cold baffle around the FPA, with a partial array opaque mask (in the past, with proper, cold, dark baffles we have achieved light leaks $<0.002 e^{-} / s$ for InSb SCAs). Therefore we are confident that the dark current at temperatures below $38 \mathrm{~K}$ is not limited by a light leak.

For comparison, we list measured dark currents at $\mathrm{T}=15 \mathrm{~K}$ and $\mathrm{T}=30 \mathrm{~K}$ for several $\mathrm{InSb}$ detector arrays in Table 3. We predict that the two InSb detector arrays in IRAC will still meet the original science driven requirement of dark current less than $1 e^{-} / s$ for all temperatures less than $37 \mathrm{~K}$ (recall predicted post-cryogen final temperature is approximately $28 \mathrm{~K}$ ).

Table 3. Data from InSb arrays bonded to CRC-744 ROICs (i.e. SCAs similar to those used in IRAC).

\begin{tabular}{|c|c|c|c|c|c|c|c|c|}
\hline & & $\begin{array}{c}\text { Channel } 1^{\dagger} \\
48534\end{array}$ & $\begin{array}{c}\text { Channel } 2^{\dagger} \\
48975\end{array}$ & 48538 & 422574 & 422575 & 422582 & 422583 \\
\hline $\bar{T}=15 \mathrm{~K}$ & " Dark Current $\left(e^{-} / \mathrm{s}\right)^{*}$ & 0.13 & 0.2 & $\overline{0.04}$ & $\overline{0.018}$ & $\overline{0.048}$ & 0.023 & 0.017 \\
\hline $\mathrm{T}=30 \mathrm{~K}$ & Dark Current $\left(e^{-} / \mathrm{s}\right)^{*}$ & & & 0.15 & 0.021 & 0.033 & 0.047 & 0.040 \\
\hline
\end{tabular}

\subsection{Noise}

Again, for comparison with flight detector arrays, the total noise per pixel is given in Table 4 for several InSb detector arrays operating at both $\mathrm{T}=15 \mathrm{~K}$ and $\mathrm{T}=30 \mathrm{~K}$. There is a sizable variation in total noise in Table 4 for the different SCAs tested. The main difference in total noise at $\mathrm{T}=15 \mathrm{~K}$ is due to the ROICs, where the Channel 1 and 2 and 48538 SCAs use Lot 1 while the SCAs in the 4225xx series use ROICs from Lot 4 . The Lot 4 ROICs 


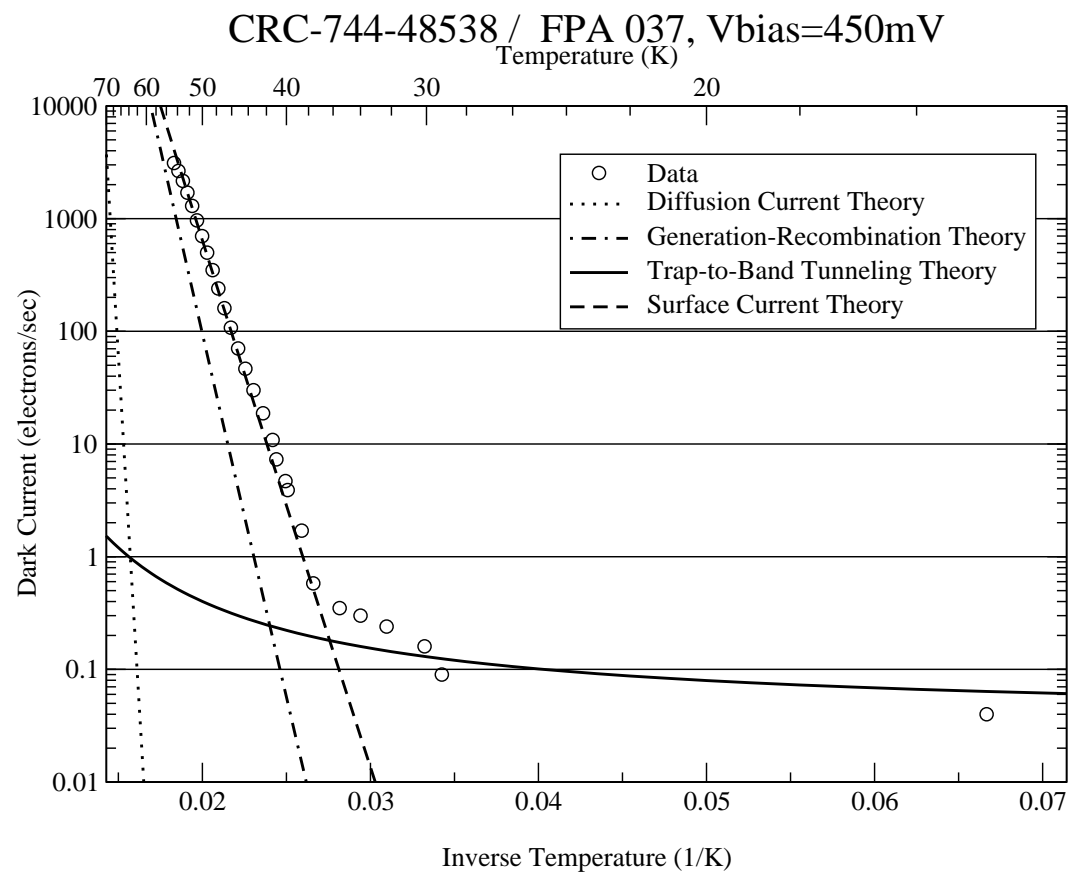

Figure 4. Arrhenius plot of dark current versus inverse temperature data for the flight mounted SCA 48538 with an applied $450 \mathrm{mV}$ detector reverse bias. Also plotted are theoretical curves for various dark current mechanisms. Notice that below about $38 \mathrm{~K}$, this SCA is limited by trap-to-band tunneling dark currents.

Table 4. Noise data from InSb arrays bonded to CRC-744 ROICs (i.e. SCAs similar to those used in IRAC).

\begin{tabular}{|c|c|c|c|c|c|c|c|c|}
\hline & & $\begin{array}{c}\text { Channel } 1 \\
48534\end{array}$ & $\begin{array}{c}\text { Channel } 2 \\
48975\end{array}$ & 48538 & 422574 & 422575 & 422582 & 422583 \\
\hline $\mathrm{T}=15 \mathrm{~K}$ & Noise $\left(e^{-}\right)^{*}$ & 8.3 & 7.3 & 7.8 & 13.3 & 12.9 & 13.6 & 12.7 \\
\hline $\mathrm{T}=30 \mathrm{~K}$ & Noise $\left(e^{-}\right)^{*}$ & & & $13.0(8.3)^{\dagger}$ & 11.7 & 12.3 & 15.1 & 12.8 \\
\hline
\end{tabular}

* The noise was obtained for a 200 second dark integration with Fowler-32 sampling, i.e. total noise includes read noise, noise from dark current and system noise. Values are per pixel.

$\dagger$ Noise in parenthesis is the total noise in a 6 second dark integration with Fowler-32 sampling.

had a processing change that resulted in the entire lot performing worse than Lot 1 ROICs. The difference in total noise between $\mathrm{T}=15 \mathrm{~K}$ and $\mathrm{T}=30 \mathrm{~K}$ for $\mathrm{SCA} 48538$ was not expected, but also not unprecedented. In addition, the total noise for SCA 48538 at $30 \mathrm{~K}$ is $8.3 e^{-}$for a 6 second Fowler-32 integration and $13 e^{-}$for a 200 second Fowler-32 integration. However, despite the apparent increase with integration time, the dark current contribution to the total noise is small compared with the read noise component at both temperatures. Typically the CRC-744 ROICs had very little change in read noise for temperatures between $14 \mathrm{~K}$ and $40 \mathrm{~K}$. We have seen a similar increase in read noise near $\mathrm{T}=30 \mathrm{~K}$ for one other $\mathrm{InSb}$ detector array, namely SCA 40716 (9e- @ $15 \mathrm{~K}$ and 14e $\left.e^{-} @ 30 \mathrm{~K}\right)$. SCA 40716 had the same Lot 1 ROIC as SCA 48538 and Channels 1 and 2. Conversely, in more recent ultra low noise ROICs (SB-226, SB-290, SB-304, SB-305), ${ }^{9,10,11}$ we have seen low noise at T=30K and higher noise at temperatures below 20K. In those modern ROICs, the higher noise at low temperatures was attributed to micro-leakage currents between row clocks and the analog ground (Vsub) within the unit cells. The micro-leakage currents are of order $50 \mu \mathrm{A}$ and directly impact the noise performance of the source follower 
FET in the unit cell due to the changing potential of the analog ground. The micro-leakage currents add to the noise power spectral density (above the white noise component, as clock feed-through) at frequencies higher than $100 \mathrm{~Hz} .{ }^{11}$ We have not directly observed such leakage currents in the CRC-744 ROICs. While it is not guaranteed that the IRAC Channel 1 and 2 SCAs will have the same noise increase as SCA 48538 from T=15K to $\mathrm{T}=30 \mathrm{~K}$, such an increase in noise will not dramatically impact the science observations. Particularly, the science observations will still be zodiacal background limited at longer integration times (100s). Further, the read noise at short integration times will also still be less than the original Spitzer requirements.

\subsection{Quantum Efficiency}

The quantum efficiency $(\mathrm{QE})$ of the InSb detector arrays will not change dramatically $(\Delta \mathrm{QE}<2 \%)$ over the temperature range of 15 - 35K. In addition, the cutoff wavelength of InSb will also not vary by a significant amount. Further, the cutoff wavelength (with slight shift) is still outside the transmission windows defined by the Channel 1 and 2 filters. Consequently, we did not measure the $\mathrm{QE}$ at $\mathrm{T}=30 \mathrm{~K}$. The quantum efficiencies are $87 \%$ and $86 \%$ for Channels 1 and 2, respectively.

\subsection{Residual Images}

Residual, persistent, latent or ghost images are the left-over signals in any subsequent (dark) image after exposure to a bright source (see Benson, et al. ${ }^{12}$ ). The original Spitzer Category III requirements on residual images are given in Table 5. Results of tests to quantify image persistence for several $\mathrm{InSb}$ detector arrays are given in Table 6. For all InSb detector arrays tested at temperatures of both $15 \mathrm{~K}$ and $30 \mathrm{~K}$, we measured larger

Table 5. Image residuals per pixel requirement for Spitzer Space Telescope IRAC InSb detector arrays.

\begin{tabular}{l|cccccc} 
& $\begin{array}{c}\text { Source } \\
\text { Flux } \\
\left(e^{-} / \mathrm{s}\right)\end{array}$ & $\begin{array}{c}\text { Source } \\
\text { Exposure } \\
(\mathrm{s})\end{array}$ & $\begin{array}{c}\text { Source } \\
\text { Fluence } \\
\left(e^{-}\right)\end{array}$ & $\begin{array}{c}\text { Delay } \\
(\mathrm{s})\end{array}$ & $\begin{array}{c}\text { Residual } \\
\text { Integration } \\
\text { Time }(\mathrm{s})\end{array}$ & $\begin{array}{c}\text { Required * } \\
\text { Residual } \\
\text { Fluence }\left(e^{-}\right)\end{array}$ \\
\hline \hline Residual Test 1 & 2500 & 20 & 50000 & 0 & 20 & 10 \\
Residual Test 2 & 2500 & 20 & 50000 & 200 & 20 & 1 \\
Residual Test 3 & 250 & 200 & 50000 & 0 & 200 & 5 \\
Residual Test 4 & 2500 & 100 & 250000 & 0 & 20 & 100 \\
Residual Test 5 & 2500 & 100 & 250000 & 200 & 20 & 10 \\
\hline
\end{tabular}

* Any residual image must be below the required residual fluence (for the given integration time) after the listed delay.

Table 6. Results from residual image tests at $\mathrm{T}=15 \mathrm{~K}$ and $\mathrm{T}=30 \mathrm{~K}$.

\begin{tabular}{cc|ccccccc} 
& & Channel 1 & Channel 2 & & & & & \\
& 48534 & 48975 & 48538 & 422574 & 422575 & 422582 & 422583 \\
\hline \hline \multirow{3}{*}{$15 \mathrm{~K}$} & Residual Test 1 $\left(e^{-}\right)^{*}$ & 72 & 25 & 68 & 10.8 & 11.5 & 11.2 & 9.1 \\
& Residual Test 2 $\left(e^{-}\right)$ & 3 & 2 & 8 & 2.0 & 0.0 & 0.0 & 0.0 \\
& Residual Test 3 $\left(e^{-}\right)$ & 168 & 6.5 & 113 & & & & \\
& Residual Test 4 $\left(e^{-}\right)$ & 360 & 23 & 422 & 94.0 & 98.5 & 87.3 & 91.8 \\
& Residual Test 5 $\left(e^{-}\right)$ & 22 & 2 & 26 & 2.8 & 2.2 & 2.4 & 0.4 \\
\hline \multirow{3}{*}{$30 \mathrm{~K}$} & & & 1220 & 117 & 129 & 313 & 287 \\
& Residual Test 1 $\left(e^{-}\right)$ & & 0 & 3.7 & 2.1 & 2.2 & 6.7 \\
& Residual Test 2 $\left(e^{-}\right)$ & & & & & & & \\
& Residual Test 3 $\left(e^{-}\right)$ & & & 2560 & 632 & 628 & 1133 & 1174 \\
& Residual Test 4 $\left(e^{-}\right)$ & & 0 & 7.5 & 6.5 & 28.5 & 25.5 \\
\hline
\end{tabular}

* Values are per pixel. There is $\pm 3 e^{-}$uncertainty on all Residual Test measurements. 
magnitude residual images at $30 \mathrm{~K}$ versus those at $15 \mathrm{~K}$ for the short time scale Tests 1 and 4 . Further we noted that the residual images decayed away more rapidly at higher temperatures. At $30 \mathrm{~K}$ the residual images were below the noise after 80 seconds ( 4 resets and 4 full frame images at 20 seconds integration), whereas at $15 \mathrm{~K}$, the residual images had decayed to less than the noise after 140 seconds $(7$ resets and full frame images at 20 seconds integration). The deviations in both magnitude and decay rates for the two temperatures are consistent with variations in the densities and spatial locations of residual causing traps, ${ }^{12}$ i.e. a given type of trap (specific impurity) may not be excited at low temperature, while at higher temperatures that same trap may dominate the residual behavior of all trap types. While the residual image performance in Tests 1 and 4 for SCA 48538 (detector array most similar to the IRAC detector arrays) is around an order of magnitude worse at 30K than at $15 \mathrm{~K}$, this does not preclude operation of the InSb detector arrays in the post cryogen era, but does indicate the need for filtering of dithered image data to remove the residual image, as is already done for cosmic ray hits and stray light. The larger initial but more rapidly decaying residual images at $\mathrm{T}=30 \mathrm{~K}$ versus $\mathrm{T}=15 \mathrm{~K}$ should not prove detrimental to Spitzer post cryogen science observations.

Another form of persistent images, called long lived residuals, is found in both Channels 1 and 4 of the IRAC instrument. ${ }^{3}$ These long lived residuals are low level persistent images that can last for days or weeks. The current mode of operation on IRAC requires an anneal every 12 hours for the Channel $1 \mathrm{SCA}$ to $\mathrm{T}=23 \mathrm{~K}$ to remove the long lived residuals. The long lived residuals are caused by a broken clamp circuit for Channel 1 in the IRAC electronics, combined with exposure to high saturating flux levels. As we have shown in previous reports ${ }^{3}$ the ROIC lot split along with a broken clamp circuit is vital to reproducing long lived residuals in our laboratory testing of sister parts to Channel 1. Since SCA 48538 has a very similar ROIC to that of Channel 1 , we were able to reproduce long lived residuals at both $\mathrm{T}=15 \mathrm{~K}$ and $\mathrm{T}=30 \mathrm{~K}$ in SCA 48538 by turning off the clamp circuit and exposing the detector array to a saturating flux. However, based upon the current IRAC anneal to $\mathrm{T}=23 \mathrm{~K}$, we had initially predicted that there would be no long lived residuals at $\mathrm{T}=30 \mathrm{~K}$. While this prediction did not prove correct, the levels of the long lived residuals at $\mathrm{T}=30 \mathrm{~K}$ are greatly reduced. The fluence of the long lived residuals at $30 \mathrm{~K}$ were $14 e^{-}$in a 20 second Fowler-32 sampled image, whereas the long lived residuals had a fluence of $39 e^{-}$at $15 \mathrm{~K}$.

\subsection{Black Holes}

Since the early days (mid 1980s) of InSb infrared detector arrays, an anomaly has been intermittently detected in images from a wide variety of devices. The anomaly is called a "black hole" and is characterized by a circular region of negative signal surrounded by a diamond-shaped region (square rotated by $45^{\circ}$ ) with positive signal (see Figure 5). The profile of the circular negative region is Gaussian, with the center being most negative. Some variation in spatial extent of the "black holes" has been seen, but they do tend to be around 20 pixel diameter at FWHM, independent of signal strength. The centers of the "black holes" (minima of inverted Gaussians) ranged between $-10 e^{-}$(near noise limit) to $-2000 e^{-}$. The average of the negative and positive signal over the entire affected area is equal to the average of the nearby background, i.e. the light (positive) and dark (negative) area are redistributions of charge and no net charge is measured. "Black holes" may appear as a single occurrence in an image, as multiple separate instances or as multiple overlapping instances. We have seen the "black holes" in images taken at temperatures between $25 \mathrm{~K}$ and $45 \mathrm{~K}$, but not at temperatures below $25 \mathrm{~K}$. Typically a "black hole" will appear in an image where the temperature of the SCA is drifting at a rate between $0.05 \mathrm{~K} / \mathrm{min}$ and $1.0 \mathrm{~K} / \mathrm{min}$. The "black holes" appeared in image data taken with several generations of InSb detector arrays from Raytheon (SBRC): in CRC-228 $(58 \times 62$ pixels), CRC-463 $(256 \times 256$ pixels $)$, CRC-744 $(256 \times 256$ pixels, Spitzer model) and SB-226 $(1024 \times 1024$ pixels $)$, but have not been detected in the SB-304 $(2048 \times 2048$ pixels $)$ InSb detector arrays. The origin of the "black holes" is not understood. The "black holes" do not appear to be associated with cosmic ray hits, since the rate at which "black holes" appears for a given device is much more variable than the variations in the cosmic ray flux. Further, most images taken in our laboratory do not show "black holes", yet all the data do have cosmic ray hits. We believe that the likely cause of "black holes" is the changing temperature which induces a temperature gradient in the InSb crystal. That temperature gradient can create stress in the crystal lattice which when released will produce phonons (and/or excitons, plasmons). ${ }^{13,14}$ The initial release of energy causes electron-hole pair production. In the "black hole" or negative region, electrons are concentrated in a Gaussian profile at the locus of the lattice slip and further add to the reverse bias. The usual creation of electron-hole pairs occurs in the bulk material where the holes are then collected at the p-n 


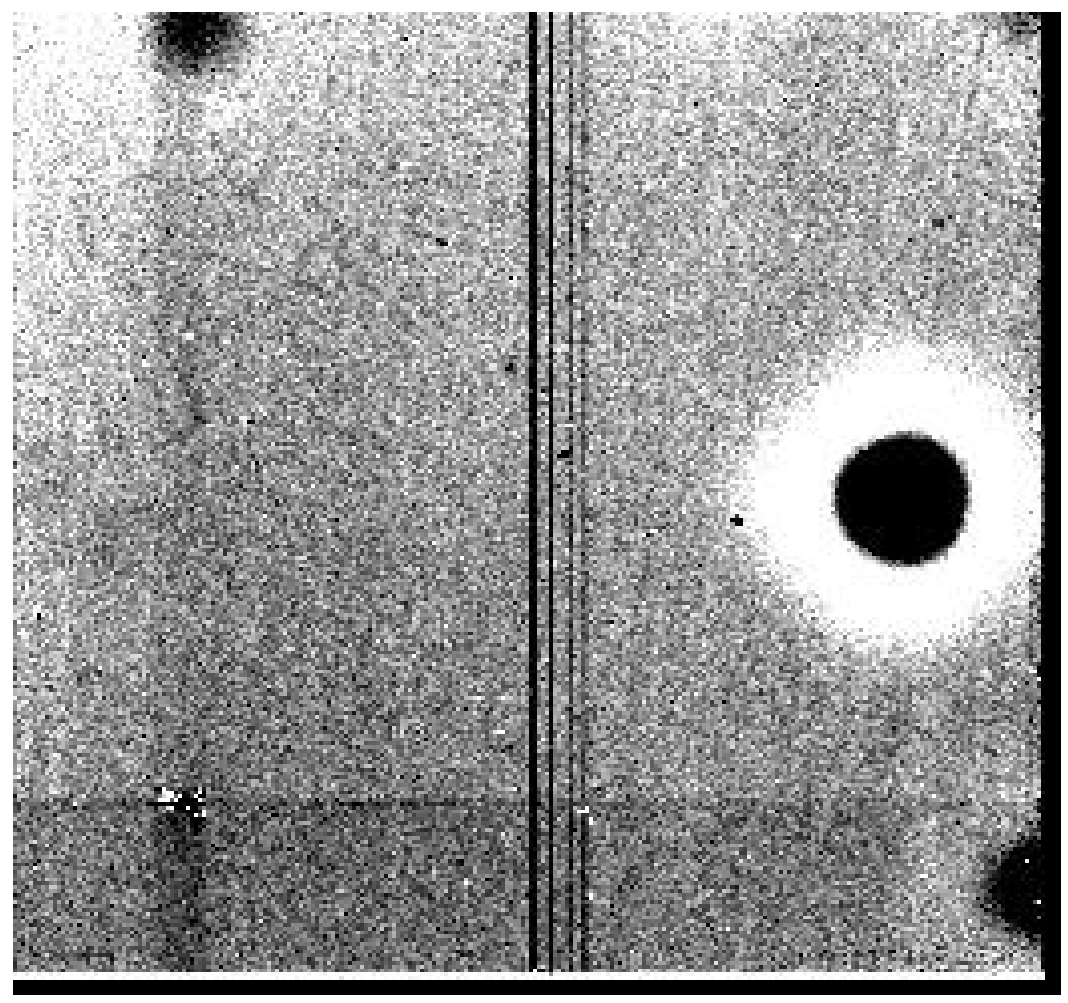

Figure 5. This image from SCA 41626 shows the phenomena called "black holes". There are two "black holes" in this image: 1) a strong signal "black hole" located mid-way from the top to bottom and near the right side, and 2) a weak signal "black hole" located near the lower right corner. The "black holes" are characterized by circular region of negative signal (black) surrounded by a diamond-shaped region of positive signal (white).

junction and the electrons are removed through the detector substrate voltage. Thus, the phonon production must occur near the surface of the InSb that is closest to the ROIC (also where greatest stress in the InSb crystal is caused by the bump bonds) and therefore allow the electrons to be collected at the p-n junction and accelerate the holes outward from the region. The white regions are pixels where holes have been collected and debias the individual detectors. The propagation of the energy (phonons or plasmons) may depart from the original Gaussian shaped "black hole" due to influences by the InSb crystal or detector processing or the numerous bump bonds to the ROIC to create the white diamond pattern, which is oriented along rows and columns.

During the measurements of all of the above quantities for SCA 48538, we obtained and stored numerous images. Some data sets contain over 200 hours of continuous operation of the SCA. In all the data obtained at stabilized temperatures near 30K, we did not observe a single "black hole" for SCA 48538. Further, we introduced a temperature rate of change of $0.6 \mathrm{~K} / \mathrm{min}$ and took image data from $25 \mathrm{~K}$ to $55 \mathrm{~K}$ and again did not find any "black holes".

Prior to the launch of the Spitzer Space Telescope, ground based laboratory testing on SCA 48975 (IRAC Channel 2) demonstrated the existence of "black holes" for all temperatures between $26.9 \mathrm{~K}$ and $42.8 \mathrm{~K}$, where the temperature was increased at a rate of $0.45 \mathrm{~K} / \mathrm{min}$ starting from $15 \mathrm{~K}$ and ending at $60 \mathrm{~K}$.

No "black holes" have been observed in InSb detector arrays where the temperature has been stabilized. We do expect the InSb detector arrays on the Spitzer Space Telescope to show "black holes" when the temperature is changing by greater than $0.1 \mathrm{~K} / \mathrm{min}$. However, we have shown that the flight mounted FPAs, operating under standard IRAC observing modes, are quite thermally stable even without active temperature control. Thus we believe that IRAC InSb Channels 1 and 2 will not have "black holes" in science data. 


\section{CONCLUSIONS}

The InSb detector arrays (Channels 1 and 2) of the IRAC instrument on the Spitzer Space Telescope will continue to operate after the liquid helium cryogen is exhausted. The IRAC instrument will equilibrate near 30K. Compared with present flight operation at $15 \mathrm{~K}$, at the final higher temperature, the InSb arrays will perform with no change in most parameters (QE, gain, capacitance), a modest degradation in both dark current and noise, and a significant worsening of residual images. As discussed above, the residual images are mitigated by the use of filtering during image processing on the ground. The slightly higher dark current and noise are still within acceptable limits. Observations will be zodiacal background limited. We have shown that the InSb detector arrays on the Spitzer Space Telescope will continue to perform within the original science driven instrument requirements after the cryogens run out.

\section{ACKNOWLEDGMENTS}

Funding for this work was provided by: Phase E Engineering Support for Instrument Operations of the Infrared Array Camera (IRAC) for the Spitzer Space Telescope, Award No. SV4-74019.

\section{REFERENCES}

1. G. G. Fazio, J. L. Hora, L. E. Allen, M. L. N. Ashby, P. Barmby, L. K. Deutsch, J. S. Huang, S. Kleiner, M. Marengo, S. T. Megeath, G. J. Melnick, M. A. Pahre, B. M. Patten, J. Polizotti, H. A. Smith, R. S. Taylor, Z. Wang, S. P. Willner, W. F. Hoffmann, J. L. Pipher, W. J. Forrest, C. W. McMurtry, C. R. McCreight, M. E. McKelvey, R. E. McMurray, D. G. Koch, S. H. Moseley, R. G. Arendt, J. E. Mentzell, C. T. Marx, P. Losch, P. Mayman, W. Eichhorn, D. Krebs, M. Jhabvala, D. Y. Gezari, D. J. Fixsen, J. Flores, K. Shakoorzadeh, R. Jungo, C. Hakun, L. Workman, G. Karpati, R. Kichak, R. Whitley, S. Mann, E. V. Tollestrup, P. Eisenhardt, D. Stern, V. Gorjian, B. Bhattacharya, S. Carey, B. O. Nelson, W. J. Glaccum, M. Lacy, P. J. Lowrance, S. Laine, W. T. Reach, J. A. Stauffer, J. A. Surace, G. Wilson, E. L. Wright, A. Hoffman, G. Domingo, and M. Cohen, "The Infrared Array Camera (IRAC) for the Spitzer Space Telescope," Astrophysical Journal, Supp. Series 154, pp. 10-17, 2004.

2. J. L. Hora, G. G. Fazio, L. E. Allen, M. L. N. Ashby, P. Barmby, L. K. Deutsch, J. S. Huang, S. Kleiner, M. Marengo, S. Megeath, G. J. Melnick, M. A. Pahre, B. M. Patten, H. A. Smith, Z. Wang, S. P. Willner, W. F. Hoffmann, J. L. Pipher, W. J. Forrest, C. W. McMurtry, C. R. McCreight, M. E. McKelvey, R. E. McMurray, D. G. Koch, S. H. Moseley, R. G. Arendt, J. E. Mentzell, C. T. Marx, D. J. Fixsen, E. V. Tollestrup, P. Eisenhardt, D. Stern, V. Gorjian, B. Bhattacharya, S. Carey, W. J. Glaccum, M. Lacy, P. Lowrance, S. Laine, B. O. Nelson, W. T. Reach, J. A. Stauffer, J. A. Surace, G. Wilson, and E. Wright, "In-flight performance and calibration of the IRAC for the Spitzer Space Telescope," in Proc. SPIE, Optical, Infrared, and Millimeter Space Telescopes, J. C. Mather, ed., 5487, pp. 77-92, 2004.

3. J. Pipher, C. McMurtry, W. Forrest, C. McCreight, M. McKelvey, R. McMurray, R. Johnson, G. Fazio, J. Hora, L. Allen, M. Ashby, P. Barmby, L. Deutsch, J. Huang, M. Marengo, T. Megeath, M. Pahre, B. Patten, Z. Wang, S. Willner, W. Hoffmann, H. Moseley, R. Arendt, D. Krebs, P. Eisenhardt, D. Stern, V. Gorjian, B. Bhattacharya, W. Glaccum, M. Lacy, P. Lowrance, S. Carey, S. Laine, J. Stauffer, J. Surace, W. Reach, and G. Wilson, "Comparison of laboratory and inflight performance of IRAC detector arrays on Spitzer Space Telescope," in Proc. SPIE, Optical, Infrared, and Millimeter Space Telescopes, J. C. Mather, ed., 5487, p. 235, 2004.

4. J. L. Hora, G. G. Fazio, S. P. Willner, M. L. Ashby, J. Huang, S. T. Megeath, J. R. Stauffer, E. V. Tollestrup, Z. Wang, W. J. Glaccum, J. L. Pipher, W. J. Forrest, C. R. McCreight, M. E. McKelvey, W. F. Hoffman, P. Eisenhardt, J. A. Surace, W. T. Reach, S. H. Moseley, R. G. Arendt, K. P. Stewart, and F. D. Robinson, "Calibration and performance of the infrared array camera (IRAC)," in Proc. SPIE, Infrared Spaceborne Remote Sensing VIII, M. Strojnik and B. F. Andresen, eds., 4131, pp. 13-25, 2000.

5. J. L. Pipher, W. J. Forrest, W. J. Glaccum, R. G. Benson, D. J. Krebs, M. D. Jhabvala, J. P. Rosbeck, N. A. Lum, W. Y. Lum, J. D. Garnett, A. W. Hoffman, G. Domingo, G. M. Cushman, and D. A. Rapchun, "InSb arrays for IRAC (infrared array camera) on SIRTF (Space Infrared Telescope Facility)," in Proc. SPIE, Infrared Spaceborne Remote Sensing VIII, M. Strojnik and B. F. Andresen, eds., 4131, pp. 7-12, 2000. 
6. G. G. Fazio, J. L. Hora, S. P. Willner, J. R. Stauffer, M. L. Ashby, Z. Wang, E. V. Tollestrup, J. L. Pipher, W. J. Forrest, C. R. McCreight, S. H. Moseley, W. F. Hoffmann, P. Eisenhardt, and E. L. Wright, "Infrared array camera (IRAC) for the Space Infrared Telescope Facility (SIRTF)," in Proc. SPIE, Infrared Astronomical Instrumentation, A. M. Fowler, ed., 3354, pp. 1024-1031, 1998.

7. J. Wu, W. J. Forrest, J. L. Pipher, N. Lum, and A. Hoffman, "Development of infrared focal plane arrays for space," Review of Scientific Instruments 68, pp. 3566-3578, 1997.

8. C. M. Bacon, C. W. McMurtry, J. L. Pipher, W. J. Forrest, and J. D. Garnett, "Burst noise in the HAWAII1RG multiplexer," in Proc. SPIE, Focal Plane Arrays for Space Telescopes II, T. J. Grycewicz and C. J. Marshall, eds., 5902, pp. K1-K12, 2005.

9. C. W. McMurtry, W. J. Forrest, A. C. Moore, and J. L. Pipher, "Next Generation Space Telescope: NIR InSb Array Development," in Proc. SPIE, IR Space Telescopes and Instruments, J. C. Mather, ed., 4850, pp. 847-857, 2002.

10. C. W. McMurtry, W. J. Forrest, A. C. Moore, and J. L. Pipher, "James Webb Space Telescope: Characterization of Flight Candidate NIR InSb Detector Arrays," in Proc. SPIE, Focal Plane Arrays for Space Telescopes, T. J. Grycewicz and C. R. McCreight, eds., 5167, pp. 144-158, 2003.

11. C. W. McMurtry, W. J. Forrest, and J. L. Pipher, "James Webb Space Telescope: Noise Results for the Multiplexers of the Mid-Infrared Instrument (MIRI)," in Proc. SPIE, Focal Plane Arrays for Space Telescopes II, T. J. Grycewicz and C. J. Marshall, eds., 5902, pp. 59028-1 - 59028-13, 2005.

12. R. G. Benson, W. J. Forrest, J. L. Pipher, W. J. Glaccum, and S. L. Solomon, "Spatial distributions of hole traps and image latency in InSb focal plane arrays," in Proc. SPIE, Infrared Spaceborne Remote Sensing VIII, M. Strojnik and B. F. Andresen, eds., 4131, pp. 171-184, 2000.

13. C. Kittel, Introduction to Solid State Physics, New York: Wiley, 7th ed., 1996.

14. S. E. Hebboul and J. P. Wolfe, "Imaging of large-k phonons in InSb," Phys Rev B 34, pp. 3948-3960, Sept. 1986. 